MATHEMATICS OF COMPUTATION

Volume 70, Number 233, Pages 319-327

S 0025-5718(00)01194-7

Article electronically published on March 1, 2000

\title{
ON SIMPLE DOUBLE ZEROS AND BADLY CONDITIONED ZEROS OF ANALYTIC FUNCTIONS OF $n$ VARIABLES
}

\author{
JEAN-PIERRE DEDIEU AND MIKE SHUB
}

Abstract. We give a numerical criterion for a badly conditioned zero of a system of analytic equations to be part of a cluster of two zeros.

\section{INTRODUCTION AND MAIN RESULTS}

Let $f: \mathbb{C}^{n} \rightarrow \mathbb{C}^{n}$ be analytic. An isolated zero of multiplicity $m$ for $f$ is a point $x \in \mathbb{C}^{n}$ such that

(1) $f(x)=0$,

(2) there exists a ball $B$ containing $x$ such that $x$ is the only zero of $f$ in $B$, and

(3) a generic analytic $g$ sufficiently close to $f$ has $m$ simple zeros $x_{1}, \ldots, x_{m}$ in $B$.

In (3) any analytic $g$ sufficiently close to $f$ such that

(4a) $\|f(y)-g(y)\|<\|f(y)\|$ for all $y \in \partial B$, where $\partial B$ is the boundary of the ball, and such that

(4b) 0 is a regular value of $g: B \rightarrow \mathbb{C}^{n}$

will do.

In fact, any analytic $g$ satisfying (4a) has a finite number of zeros in $B$ and the sum of the multiplicities of these zeros is $m$. This fact is known as Rouché's Theorem in the literature, see Berenstein et al. [2], Theorem 2.12.

The number $m$ can be computed from homology theory. It is the degree of the mapping

$$
f_{*}: H_{2 n-1}(B, \partial B) \rightarrow H_{2 n-1}\left(\mathbb{C}^{n}, \mathbb{C}^{n} \backslash\{0\}\right) .
$$

Any map satisfying (4a) induces the same map as $f$ on homology and has the same degree. For an analytic $g$ such that 0 is a regular value, the degree of

$$
g_{*}: H_{2 n-1}(B, \partial B) \rightarrow H_{2 n-1}\left(\mathbb{C}^{n}, \mathbb{C}^{n} \backslash\{0\}\right)
$$

is the sum of the signs of the Jacobian determinants at the zeros $x_{1}, \ldots, x_{m}$ of $g$. But as $g$ is complex analytic, each of these signs is +1 .

The multiplicity $m$ of $x$ may also be computed as the dimension of the local algebra of $f$ at $x$, see Arnold et al. [1]. Consider the local algebra $\mathbb{C}\{z\}_{x}$ of all holomorphic function-germs at $x$. The germs of the components of $f$ generate an

Received by the editor January 12, 1999.

2000 Mathematics Subject Classification. Primary 65H10.

Key words and phrases. Systems of equations, multiple zeros, condition numbers.

This work was done while both authors were at MSRI, Berkeley, in fall 1998, for the Foundations of Computational Mathematics program.

Partially supported by the National Science Foundation. 
ideal $I_{f, x}$ in this algebra. The multiplicity of $f$ at $x$ is the dimension of its local algebra

$$
m=\operatorname{dim}_{\mathbb{C}} \mathbb{C}\{z\}_{x} / I_{f, x},
$$

which is at least one.

A point $x$ of multiplicity $m>1$ must necessarily be a singular point of $f$, i.e., the derivative $D f(x)$ has rank less than $n$. It follows that if $g$ is sufficently close to $f$, then the $m$ zeros of $g$ must be arbitrarily badly conditioned, i.e., $\left\|D g\left(x_{i}\right)^{-1}\right\| \rightarrow \infty$ for all $i$ as $g \rightarrow f$.

We would like to be able to proceed in the opposite direction; that is, given a point $x$ such that $\|g(x)\|$ is small (or yet even $g(x)=0$ ), $D g(x)$ badly conditioned and some additional information about $g$, we would like to conclude that $x$ is close to (or part of) a cluster of $m$ zeros in a disk of radius $r$ and that the pair $(g, x)$ is close to a pair $(\tilde{g}, \tilde{x})$ such that $\tilde{x}$ is a zero of multiplicity $m$ of $\tilde{g}$. For $m=2$ we accomplish this in Theorem 4.

Besides the intrinsic interest of this problem, we are motivated by the algorithms for finding zeros of complex polynomials in one variable, which have excellent complexity estimates and which exploit zeros of higher derivatives as organizing points for clusters of zeros. The first of these to come to our attention was due to Renegar 10]; see Pan [9] for a survey. Both in one variable and in $n$ variables, Newton's method has difficulty when zeros are badly conditioned; see Smale [17, Shub-Smale [12]-16], Dedieu-Shub [5], 6], and Blum-Cucker-Shub-Smale [4]. Even in $n$ dimensions we may look for a well conditioned zero of high multiplicity for a nearby system which can serve as an organizing point for a cluster.

In this paper we will be concerned with certain points of multiplicity two, which we call simple double zeros.

Let $f: \mathbb{C}^{n} \rightarrow \mathbb{C}^{n}$ be analytic and suppose $f(x)=0$. Then $x$ is a simple double zero of $f$ if

$$
\operatorname{dim} \operatorname{ker} D f(x)=1,
$$

ker $D f(x)$ is the space spanned by the vector $v \in \mathbb{C}^{n}$ with $\|v\|=1$ and

$$
D^{2} f(x)(v, v) \notin \operatorname{im} D f(x) .
$$

In the nomenclature of singularity theory (dating from Roger [11, or see Arnold et al. 1] or Golubitsky-Guillemin [7]), condition A makes $x$ an $S_{1}$ singularity and condition B makes $x$ an $S_{1,0}$ singularity in the Thom-Boardman classification. For generic $f$, the $S_{1,0}$ singularities comprise the highest dimensional component of the singular set of $f$, and all zeros of $f$ which are also critical points of $f$ are $S_{1,0}$ singularities. It follows that a simple double zero has multiplicity 2 ; see Levine [8], Arnold et al. 1] or Golubitsky-Guillemin [7].

Given $x, v \in \mathbb{C}^{n}, v \neq 0$, we define the linear operator

$$
A(f, x, v)=D f(x) .+\frac{1}{2} D^{2} f(x)\left(v, \Pi_{v}\right) .
$$

Here $\Pi_{E}$ denotes Hermitian projection onto the subspace $E \subset \mathbb{C}^{n}$. By abusing notation slightly we have written $\Pi_{v}$ instead of $\Pi_{[v]}$, where $[v]$ is the subspace spanned by $v$. When $x$ is a simple double zero we simply write $A(f, x)$ and understand that $v$ is a unit vector in the kernel of $D f(x)$.

The linear operator $A(f, x)$ is not intrinsically defined in the sense of singularity theory; that is, it is not coordinate independent. Yet $A(f, x)$ and an associated 
quantity $\gamma_{2}$ are the focus of our analysis. When $x$ is a simple double zero, it follows then from $\mathrm{A}$ and $\mathrm{B}$ that $A(f, x)$ is invertible.

Definition 1. If $A(f, x)$ is invertible, we define

$$
\gamma_{2}(f, x)=\max \left(1, \sup _{k \geq 2}\left\|A(f, x)^{-1} \frac{D^{k} f(x)}{k !}\right\|^{1 /(k-1)}\right)
$$

and infinity otherwise.

We use the subscript 2 to distinguish $\gamma_{2}(f, x)$ from Smale's

$$
\gamma(f, x)=\sup _{k \geq 2}\left\|D f(x)^{-1} \frac{D^{k} f(x)}{k !}\right\|^{1 /(k-1)},
$$

which is defined if $D f(x)$ is invertible, and to indicate the dependence of $A(f, x)$ and hence $\gamma_{2}(f, x)$ on the second derivative of $f$. Smale introduced $\gamma(f, x)$ in [17] in the study of simple zeros of analytic functions between Banach spaces and the convergence of Newton's method towards them. If $x$ is a simple zero of $f$, then any point in the ball of radius $\frac{5-\sqrt{17}}{4 \gamma(f, x)}$ around $x$ converges very rapidly (quadratically) under Newton iteration to $x$. Thus, in particular, if $y$ is another zero of $f$, we may separate $y$ from $x$ by

$$
\|y-x\| \geq \frac{5-\sqrt{17}}{4 \gamma(f, x)}>\frac{0.21922 \ldots}{\gamma(f, x)}
$$

(see Blum-Cucker-Shub-Smale [3] or Dedieu 4], which gives the even better value $\left.\|y-x\| \geq \frac{1}{2 \gamma(f, x)}\right)$.

Here we prove a similar result for separating simple double zeros from the other zeros of $f$. In this paper $c$ is a universal constant, $c=0.19830 \ldots$.

Theorem 1. If $x$ is a simple double zero of $f$ and $y$ is another zero, then

$$
\|y-x\| \geq \frac{c}{\gamma_{2}(f, x)}
$$

The proof of Theorem 1 is easily adapted to give an estimate of $\|f(y)\|$ for any $y$ in a neighborhood of $x$.

Theorem 2. If $x$ is a simple double zero of $f$ and $\|y-x\| \leq \frac{c}{2 \gamma_{2}(f, x)^{2}}$, then

$$
\|f(y)\| \geq \frac{c\|y-x\|^{2}}{\left\|A(f, x)^{-1}\right\|} .
$$

We now use Theorem 2 to see that analytic $g$ near $f$ have zeros near $x$ such that the sum of their multiplicities is two.

For $R>0$ define

$$
d_{R}(f, g)=\max _{\|y-x\| \leq R}\|f(y)-g(y)\| .
$$

Theorem 3. Let $x$ be a simple double zero of $f$ and

$$
0<R \leq \frac{c}{2 \gamma_{2}(f, x)^{2}} .
$$

If

$$
d_{R}(f, g)<\frac{c R^{2}}{\left\|A(f, x)^{-1}\right\|},
$$

then the sum of the multiplicities of the zeros of $g$ in $B_{R}(x)$ is two. 
Corollary 1. Let $x$ be a simple double zero of $f$ and

$$
R_{0}=\frac{c}{2 \gamma_{2}(f, x)} .
$$

Assume that

$$
d_{R_{0}}(f, g)<\frac{c R_{0}^{2}}{\left\|A(f, x)^{-1}\right\|} .
$$

Then $g$ has two zeros $y_{1}$ and $y_{2}$ in $B_{R_{0}}(x)$, and for each of these

$$
\left\|x-y_{i}\right\|^{2} \leq \frac{\left\|A(f, x)^{-1}\right\|}{c} d_{R_{0}}(f, g) .
$$

Theorem 3 has a nice application described below. Namely, if $\|f(x)\|$ is small and $\|D f(x) v\|$ is also small, then $f$ has two zeros in a neighborhood of $x$. More precisely, let $x, v \in \mathbb{C}^{n}$ be given with $\|v\|=1$ and such that

$$
\left.\operatorname{rank} D f(x)\right|_{v^{\perp}}=n-1 \text { and }\left.D^{2} f(x)(v, v) \notin \operatorname{im} D f(x)\right|_{v^{\perp}} .
$$

Let us define a linear operator $L: \mathbb{C}^{n} \rightarrow \mathbb{C}^{n}$ by $L(v)=D f(x) v$ and $L(w)=0$ for any $w \in v^{\perp}$. Then the linear operator defined by

$$
B(f, x, v)=D f(x)-L+\frac{1}{2} D^{2} f(x)\left(v, \Pi_{v} .\right)=A(f, x, v)-L
$$

is nonsingular. Let us define

$$
\gamma_{2}(f, x, v)=\max \left(1, \sup _{k \geq 2}\left\|B(f, x, v)^{-1} \frac{D^{k} f(x)}{k !}\right\|^{1 /(k-1)}\right) .
$$

Theorem 4. If

$$
\|f(x)\|+\|D f(x) v\| \frac{c}{2 \gamma_{2}(f, x, v)^{2}}<\frac{c^{3}}{4\left\|B(f, x, v)^{-1}\right\| \gamma_{2}(f, x, v)^{4}},
$$

then $f$ has two zeros (counting multiplicities) in the ball of radius $\frac{c}{2 \gamma_{2}(f, x, v)^{2}}$ around $x$.

\section{Proofs}

Let us now prove these theorems. For two nonzero vectors $v, w \in \mathbb{C}^{n}$ we define their angle by

$$
d_{P}(v, w)=\arccos \frac{|\langle v, w\rangle|}{\|v\|\|w\|} .
$$

This definition is invariant under scaling: $d_{P}(\lambda v, w)=d_{P}(v, \lambda w)=d_{P}(v, w)$ for any nonzero scalar $\lambda$.

Theorems 1 and 2 are the consequences of two inequalities. In the first one we suppose that $d_{P}(y-x, v)$ is "big" while in the second one we consider the case of "small" angles. For this reason we introduce a priori a certain angle $\theta \in] 0, \frac{\pi}{2}[$ separating the two cases. The value of $\theta$ will be made precise later.

In the following $x$ is a simple double root of $f$ and we consider a vector $y \in \mathbb{C}^{n}$, $y \neq x$. Let us denote $w=y-x$. 
Lemma 1. If $\gamma_{2}(f, x)\|w\| \leq \frac{1}{2}$ and $d_{P}(v, w) \geq \theta$, then

$$
\left\|A(f, x)^{-1} f(y)\right\| \geq\|w\| \sin \theta-2\|w\|^{2} \gamma_{2}(f, x) .
$$

Proof. By Taylor's formula

$$
\begin{aligned}
f(y) & =f(x)+D f(x) w+\sum_{k \geq 2} \frac{D^{k} f(x) w^{k}}{k !} \\
& =D f(x) w+\frac{1}{2} D^{2} f(x)\left(v, \Pi_{v} w\right)-\frac{1}{2} D^{2} f(x)\left(v, \Pi_{v} w\right)+\sum_{k \geq 2} \frac{D^{k} f(x) w^{k}}{k !} .
\end{aligned}
$$

Since $A=A(f, x)=D f(x) .+\frac{1}{2} D^{2} f(x)\left(v, \Pi_{v}.\right)$ is nonsingular and $\|v\|=1$, we get

$$
A^{-1} \frac{1}{2} D^{2} f(x)\left(v, \Pi_{v} w\right)=A^{-1} \frac{1}{2} D^{2} f(x)(v,\langle w, v\rangle v)=\langle w, v\rangle A^{-1} A v=\langle w, v\rangle v,
$$

so that

$$
A^{-1} f(y)=w-\langle w, v\rangle v+\sum_{k \geq 2} A^{-1} \frac{D^{k} f(x) w^{k}}{k !}
$$

or

$$
\Pi_{v^{\perp}} w=A^{-1} f(y)-\sum_{k \geq 2} A^{-1} \frac{D^{k} f(x) w^{k}}{k !} .
$$

By the triangle inequality we get

$$
\begin{aligned}
\|w\| \sin \theta & \leq\|w\| \sin d_{P}(v, w)=\left\|\Pi_{v^{\perp}} w\right\| \\
& \leq\left\|A^{-1} f(y)\right\|+\sum_{k \geq 2} \gamma_{2}(f, x)^{k-1}\|w\|^{k} \\
& \leq\left\|A^{-1} f(y)\right\|+\gamma_{2}(f, x)\|w\|^{2} \sum_{k \geq 0}\left(\frac{1}{2}\right)^{k},
\end{aligned}
$$

because $\gamma_{2}(f, x)\|w\| \leq 1 / 2$, and we are done.

Lemma 2. Let us define

$$
A_{\alpha}(z)=D f(x) z+\alpha D^{2} f(x)\left(v, \Pi_{v} z\right),
$$

where $\alpha$ is a nonzero scalar. Then, this operator is nonsingular, and

$$
\left\|A_{\alpha}^{-1} A_{\beta}\right\|=\max \left(1,\left|\frac{\beta}{\alpha}\right|\right) .
$$

Proof. $A_{\alpha}^{-1} A_{\beta}(w)=\alpha^{-1} \beta w$ when $w \in[v]$, and $=w$ when $w \in[v]^{\perp}$.

Lemma 3. Let us define $\omega=d_{P}(v, w)$. If $\gamma_{2}(f, x)\|w\| \leq \frac{1}{2}$, then

$$
\begin{aligned}
& \left\|A(f, x)^{-1} f(y)\right\| \\
& \quad \geq\|w\|^{2}\left(\cos \omega-2 \gamma_{2}(f, x) \cos \omega \sin \omega-\gamma_{2}(f, x) \sin ^{2} \omega-2 \gamma_{2}(f, x)^{2}\|w\|\right) .
\end{aligned}
$$


Proof. Let us write $y-x=w=\alpha v+w_{1}=\langle w, v\rangle v+w_{1}$ with $w_{1} \in v^{\perp}$. By Taylor's formula we get

$$
\begin{aligned}
f(y) & =D f(x) w+\frac{1}{2} D^{2} f(x)(\alpha v, \alpha v)-\frac{1}{2} D^{2} f(x)(\alpha v, \alpha v)+\sum_{k \geq 2} \frac{D^{k} f(x) w^{k}}{k !} \\
& =A_{\frac{\alpha}{2}} w+\alpha D^{2} f(x)\left(v, w_{1}\right)+\frac{1}{2} D^{2} f(x)\left(w_{1}, w_{1}\right)+\sum_{k \geq 3} \frac{D^{k} f(x) w^{k}}{k !}
\end{aligned}
$$

so that

$$
\begin{aligned}
A_{\frac{\alpha}{2}}^{-1} A_{\frac{1}{2}} A_{\frac{1}{2}}^{-1} f(y)= & w+\alpha A_{\frac{\alpha}{2}}^{-1} A_{\frac{1}{2}} A_{\frac{1}{2}}^{-1} D^{2} f(x)\left(v, w_{1}\right) \\
& +\frac{1}{2} A_{\frac{\alpha}{2}}^{-1} A_{\frac{1}{2}} A_{\frac{1}{2}}^{-1} D^{2} f(x)\left(w_{1}, w_{1}\right) \\
& +\sum_{k \geq 3} A_{\frac{\alpha}{2}}^{-1} A_{\frac{1}{2}} A_{\frac{1}{2}}^{-1} \frac{D^{k} f(x) w^{k}}{k !} .
\end{aligned}
$$

Notice that $|\alpha|=|\langle w, v\rangle| \leq\|w\| \leq\|w\| \gamma_{2}(f, x) \leq 1 / 2$ because $\gamma_{2}(f, x) \geq 1$. Thus by Lemma 2 we obtain

$$
\left\|A_{\frac{\alpha}{2}}^{-1} A_{\frac{1}{2}}\right\|=\frac{1}{|\alpha|} .
$$

We also notice that $A_{\frac{1}{2}}=A$. By the triangle inequality we get

$$
\begin{aligned}
\|w\| \leq & \frac{1}{|\alpha|}\left\|A^{-1} f(y)\right\|+\left\|A^{-1} D^{2} f(x)\right\|\left\|w_{1}\right\| \\
& +\frac{1}{2|\alpha|}\left\|A^{-1} D^{2} f(x)\right\|\left\|w_{1}\right\|^{2}+\frac{1}{|\alpha|} \sum_{k \geq 3}\left\|A^{-1} \frac{D^{k} f(x)}{k !}\right\|\|w\|^{k} .
\end{aligned}
$$

Using the definition of $\gamma_{2}(f, x)$ and the equalities $|\alpha|=\|w\| \cos \omega,\left\|w_{1}\right\|=\|w\| \sin \omega$, we obtain

$$
\begin{aligned}
\|w\|^{2} \cos \omega \leq & \left\|A^{-1} f(y)\right\|+2 \gamma_{2}(f, x)\|w\|^{2} \cos \omega \sin \omega \\
& +\gamma_{2}(f, x)\|w\|^{2} \sin ^{2} \omega+\sum_{k \geq 3} \gamma_{2}(f, x)^{k-1}\|w\|^{k} .
\end{aligned}
$$

This last sum is $\leq 2 \gamma_{2}(f, x)^{2}\|w\|^{3}$ because $\gamma_{2}(f, x)\|w\| \leq 1 / 2$, and we are done.

Lemma 4. Let

$$
d=\frac{1}{6}(8+6 \sqrt{78})^{1 / 3}-\frac{7}{3}(8+6 \sqrt{78})^{-1 / 3}+\frac{1}{3}=0.39660 \ldots
$$

be the positive root of the equation

$$
\sqrt{1-d^{2}}-d \sqrt{1-d^{2}}-d-d^{2}=0 .
$$

Let us define $\theta$ by

$$
\sin \theta=\frac{d}{\gamma_{2}(f, x)}
$$


Then, for any $y \in \mathbb{C}^{n}$, with $w=y-x$ and such that $\|w\| \gamma_{2}(f, x) \leq 1 / 2$, either $d_{P}(v, w) \geq \theta$ and

$$
\left\|A(f, x)^{-1} f(y)\right\| \geq 2 \gamma_{2}(f, x)\|w\|\left(\frac{\sin \theta}{2 \gamma_{2}(f, x)}-\|w\|\right),
$$

or $d_{P}(v, w) \leq \theta$ and

$$
\left\|A(f, x)^{-1} f(y)\right\| \geq 2 \gamma_{2}(f, x)^{2}\|w\|^{2}\left(\frac{\sin \theta}{2 \gamma_{2}(f, x)}-\|w\|\right) .
$$

Proof. Let us consider the estimate given by Lemma 1:

$$
\left\|A(f, x)^{-1} f(y)\right\| \geq 2 \gamma_{2}(f, x)\|w\|\left(\frac{\sin \theta}{2 \gamma_{2}(f, x)}-\|w\|\right),
$$

valid for any $y$ with $d_{P}(v, w) \geq \theta$, and the estimate given by Lemma 3 :

$$
\begin{aligned}
& \left\|A(f, x)^{-1} f(y)\right\| \\
& \quad \geq 2 \gamma_{2}(f, x)^{2}\|w\|^{2}\left(\frac{\cos \omega-2 \gamma_{2}(f, x) \cos \omega \sin \omega-\gamma_{2}(f, x) \sin ^{2} \omega}{2 \gamma_{2}(f, x)^{2}}-\|w\|\right),
\end{aligned}
$$

which is valid for any $y$. The function

$$
\cos \omega-2 \gamma_{2}(f, x) \cos \omega \sin \omega-\gamma_{2}(f, x) \sin ^{2} \omega
$$

is decreasing for $\omega \in[0, \pi / 4]$. So, for $\theta \in[0, \pi / 4]$, we have

$$
\begin{aligned}
& \left\|A(f, x)^{-1} f(y)\right\| \\
& \quad \geq 2 \gamma_{2}(f, x)^{2}\|w\|^{2}\left(\frac{\cos \theta-2 \gamma_{2}(f, x) \cos \theta \sin \theta-\gamma_{2}(f, x) \sin ^{2} \theta}{2 \gamma_{2}(f, x)^{2}}-\|w\|\right)
\end{aligned}
$$

for any $y$ with $d_{P}(v, w) \leq \theta$. If we choose $\theta \in[0, \pi / 4]$ such that

$$
\frac{\sin \theta}{2 \gamma_{2}(f, x)} \leq \frac{\cos \theta-2 \gamma_{2}(f, x) \cos \theta \sin \theta-\gamma_{2}(f, x) \sin ^{2} \theta}{2 \gamma_{2}(f, x)^{2}}
$$

then the conclusion of Lemma 4 holds. This is achieved with $\sin \theta=\frac{d}{\gamma_{2}(f, x)}$ : when we substitute this value of $\theta$ in this inequality we obtain

$$
\sqrt{1-\frac{d^{2}}{\gamma^{2}}}-2 d \sqrt{1-\frac{d^{2}}{\gamma^{2}}}-\frac{d^{2}}{\gamma}-d \geq 0
$$

for any $\gamma \geq 1$. Since this last function of $\gamma \geq 1$ is increasing for any $d \leq 1 / 2$, it is sufficient to check this inequality for $\gamma=1$, i.e.,

$$
\sqrt{1-d^{2}}-2 d \sqrt{1-d^{2}}-d^{2}-d \geq 0
$$

The best possible value for $d$ is given by the equality to 0 , and we are done.

Proof of Theorem 1. When $f(y)=0$, then, by Lemma 4, when $\|w\| \gamma_{2}(f, x) \leq 1 / 2$, we have $\|w\| \geq \frac{\sin \theta}{2 \gamma_{2}(f, x)}$ with $\sin \theta=\frac{d}{\gamma_{2}(f, x)}$. Thus

$$
\|x-y\|=\|w\| \geq \frac{d}{2 \gamma_{2}(f, x)^{2}}=\frac{c}{\gamma_{2}(f, x)^{2}} .
$$

When $\|w\| \gamma_{2}(f, x) \geq 1 / 2$ the same conclusion holds because $\gamma_{2}(f, x) \geq 1$. 
Proof of Theorem 2. It is a consequence of Lemma 4. When

$$
\|w\| \leq \frac{c}{2 \gamma_{2}(f, x)^{2}}=\frac{d}{4 \gamma_{2}(f, x)^{2}}=\frac{\sin \theta}{4 \gamma_{2}(f, x)},
$$

then, by Lemma 4 , we have the following:

$$
\left\|A^{-1} f(y)\right\| \geq 2 \gamma_{2}(f, x)^{2}\|w\|^{2} \frac{\sin \theta}{4 \gamma_{2}(f, x)}=2 \gamma_{2}(f, x)^{2}\|w\|^{2} \frac{c}{2 \gamma_{2}(f, x)^{2}}=c\|w\|^{2} .
$$

Proof of Theorem 3. Since $\|f(y)-g(y)\|<\|f(y)\|$ for any $y$ such that $\|x-y\|=R$, $f$ and $g$ induce the same map on homology on $H_{2 n-1}(B, \partial B)$ and by Rouché's Theorem have the same number of zeros inside $B_{R}(x)$, counting multiplicities. By Theorem 1, when $R<\frac{c}{2 \gamma_{2}(f, x)}$, the only root of $f$ in $B_{R}(x)$ is $x$. The number of zeros inside that ball is two.

Proof of Theorem 4. Let us define

$$
g(y)=f(y)-f(x)-L(y-x) .
$$

We have $D g(x)=D f(x)-L$ and $D^{k} g(x)=D^{k} f(x)$ for any $k \geq 2$. Moreover, $\operatorname{ker} D g(x)=[v]$ and $\operatorname{im} D g(x)=\left.\operatorname{im} D f(x)\right|_{v^{\perp}}$, so that

$$
D^{2} g(x)(v, v) \notin \operatorname{im} D g(x) .
$$

We also notice that

$$
D g(x) .+\frac{1}{2} D^{2} g(x)\left(v, \Pi_{v} .\right)=B(f, x, v)
$$

and that $\gamma_{2}(g, x)=\gamma_{2}(f, x, v)$. Theorem 4 is a consequence of Theorem 3 applied to $g$ and $f$.

\section{REFERENCES}

[1] V. I. Arnold, S. M. Gusein-Zade, A. N. Varchenko, Singularities of Differentiable Maps. Volume I. Birkhauser, 1985. MR 86f:58018

[2] C. Berenstein, R. Gay, A. Vidras, A. Yger, Residue Currents and Bézout Identities. Birkhauser, 1993. MR 94m:32006

[3] L. Blum, F. Cucker, M. Shub, S. Smale, Complexity and Real Computation, Springer Verlag (1997). MR 99a:68070

[4] J. P. Dedieu, Condition Number Analysis for Sparse Polynomial Systems, in : Fondations of Computationnal Mathematics, F. Cucker, M. Shub Eds. Springer (1997), pp. 75-101. MR 99j:62085

[5] J. P. Dedieu, M. Shub, Multihomogeneous Newton's Method. To appear in: Math. of Computation.

[6] J. P. Dedieu, M. Shub, Newton's Method for Overdetermined Systems of Equations. To appear in: Math. of Computation.

[7] M. Golubitsky, V. Guillemin, Stable Mappings and their Singularities. Springer Verlag, 1973. MR 49:6269

[8] H. Levine, The Singularities $S_{1}^{q}$, Illinois Journal of Math. 8, 152-168 (1964). MR 28:2560

[9] V. Pan, Solving a Polynomial Equation: Some History and Recent Progress, SIAM Review, 39, 187-220 (1997). MR 99b:65066

[10] J. Renegar, On the Worst-Case Arithmetic Complexity of Approximating Zeros of Polynomials. Journal of Complexity 3, 90-113 (1987). MR 89a:68107

[11] F. Roger, Sur les variétés critiques, C. R. Acad. Sci. Paris, 208, 29-31, 1939.

[12] M. Shub, S. Smale, Complexity of Bézout's Theorem I : Geometric Aspects, J. Am. Math. Soc. 6, 459-501 (1993). MR 93k:65045 
[13] M. Shub, S. Smale, Complexity of Bézout's Theorem II : Volumes and Probabilities, in : F. Eyssette, A. Galligo Eds. Computational Algebraic Geometry, Progress in Mathematics. Vol. 109, Birkhäuser, (1993), pp. 267-285. MR 94m:68086

[14] M. Shub, S. Smale, Complexity of Bézout's Theorem III: Condition Number and Packing, J. of Complexity, 9, 4-14 (1993). MR 94g:65152

[15] M. Shub, S. Smale, Complexity of Bézout's Theorem IV : Probability of Success, Extensions, SIAM J. Numer. Anal., 33, 128-148 (1996). MR 97k:65310

[16] M. Shub, S. Smale, Complexity of Bézout's Theorem V : Polynomial Time, Theoretical Computer Science, 133, 141-164 (1994). MR 96d:65091

[17] S. Smale, Newton's Method Estimates from Data at One Point, in : The Merging of Disciplines : New Directions in Pure, Applied and Computational Mathematics, R. Ewing, K. Gross, C. Martin Eds., Springer (1986), pp. 185-196. MR 88e:65076

Laboratoire Approximation et Optimisation, Université Paul Sabatier, 31062 TouLouse Cedex 04, France

E-mail address: dedieu@cict.fr

iBM T.J. Watson Research Center, Yorktowns Heights, New York 10598-0218

E-mail address: mshub@us.ibm.com 